\title{
¿Contar sindicalistas o las sindicalistas cuentan? Discusiones sobre representación e igualdad de género en los sindicatos
}

\author{
Reseña de: Cécile Guillaume, Syndiquées: defendre les intérêts des femmes au travail, Paris, Presses de \\ Sciences Po, 2018, 254 págs. \\ Victoria Estermann \\ Centro de Investigaciones Socio-históricas (CISH)-Instituto de Investigaciones en Humanidades y Ciencias Sociales \\ (IdIHCS) - Facultad de Humanidades y Ciencias de la Educación - Universidad Nacional de La Plata; \\ Institut für Politik- und Verwaltungswissenschaften, Universidad de Rostock, Argentina \\ vickyestermann_91@hotmail.com
}

El libro de Guillaume renueva los estudios sobre sindicalismo y género en el ámbito francés, que tuvieron su auge entre 1970 y 1980, pero que luego perdieron relevancia. La autora dedica una buena parte de su libro a reseñar los trabajos sobre esta temática señalando esta ausencia. Asimismo se enfoca en destacar a las autoras británicas quienes lograron una mayor teorización y producción en este campo. En esta obra se intenta, a través del análisis de un extenso trabajo de campo, poner en tensión y debatir algunas cuestiones relacionadas con el sindicalismo y el género.

El texto se divide en tres capítulos, en el primero se dedica a realizar el estado de la cuestión sobre sindicalismo y género en los dos países que analiza: Gran Bretaña y Francia. Señala el desarrollo temprano que tuvieron los estudios de sindicalismo y género en Gran Bretaña, destaca la importancia de los trabajos pioneros ${ }^{1}$ y reseña las investigaciones actuales en Francia.

Realiza un recorrido a través de la historia de los sindicatos a analizar (CFDT ${ }^{2}$ y Solidaires ${ }^{3}$ para Francia y $\mathrm{GMB}^{4}$ y UNISON ${ }^{5}$ para Gran Bretaña) y explica cómo se fueron incorporando la perspectiva y las luchas de género al interior de estas instituciones. Expone el marco teórico y la metodología de su trabajo. En esta parte se pregunta el porqué, el qué y el cómo comparar el sindicalismo en ambos países, así como la preocupación por el abordaje empírico. La pregunta más interesante que se hace Guillaume es si para avanzar en la igualdad al interior del sindicato es solo necesario tener más mujeres en puestos de decisión (representación estadística), o también desarrollar medidas que hagan la diferencia (representación descriptiva).

Para quienes investigamos estos temas, esta pregunta resulta de vital importancia.

El segundo capítulo es el desarrollo de todo el análisis comparado de su investigación. En tres apartados (afiliarse y mantenerse afiliado, llegar a un mandato local, devenir responsable sindical) la autora explica, categoriza y ejemplifica cómo se construyen las carreras sindicales en el interior de las organizaciones analizadas, marcando diferencias por país, edad, género y estabilidad laboral. Uno de los conceptos más importantes que retoma es el de regímenes de inequidad de Joan Acker, que da cuenta de cómo las organizaciones mismas también fabrican y reproducen las desigualdades de género.

En "Afiliarse y mantenerse afiliado" hace un recorrido por las trayectorias de las sindicalistas, viendo cómo se acercaron, y por qué aún continúan participando. La autora ve que es importante la coherencia entre lo que se piensa y lo que se hace, como forma de justificación de esta militancia. No obstante, reconoce tres causas principales de la adhesión: un involucramiento progresivo con las tareas sindicales, la participación en 
las grandes huelgas de la época y, por último, cuestiones más individuales, como problemáticas en su empresa o sector.

En "llegar a un mandato local" se analiza la construcción de legitimidad y apoyo por parte de las sindicalistas, quienes ven en el mandato local una forma de justificarse ante los trabajadores. El análisis de los regímenes de inequidad que cada sindicato posee es muy importante. Aquí la autora plantea diferencias entre Gran Bretaña y Francia: en el primer país la rotación de los sindicalistas es menor, ya que permanecen por mucho tiempo y dificultan a las mujeres el acceso a los puestos más altos. En Francia, al contario, por la escasez de dirigentes, las mujeres pueden involucrarse más rápidamente en los puestos locales e intermedios. No obstante, en ambos países se señala que éstas deben "hacerse espacio" en un mundo de hombres y jugar según reglas masculinas.

Por último, el apartado de "devenir responsable sindical", explica cómo son los procesos de afirmación en la estructura sindical de ambos países. En Gran Bretaña, por ejemplo, hay dos tipos de militante sindical, el elegido democráticamente y quien ingresa como trabajador por contrato. En Francia, por el contrario, se da por cúmulo de mandatos, el/la sindicalista va ocupando distintas responsabilidades que le dan legitimidad y experiencia. No obstante también se evidencia un proceso de reclutamiento informal, desfavorable para las mujeres.

Algo que la autora destaca es la tensión entre la lucha por medidas de acción positiva, pero la no utilización de las mismas, por miedo a la pérdida de legitimidad. Esta última parte es central a la hora de analizar el sindicalismo en clave de género. La conciencia de que las medidas de cupo son necesarias para romper el techo de cristal, pero el mandato interno de no utilizarlas para que se las valore por su capacidad y no por ser dirigentes "de cupo" se presenta en la mayoría de los espacios de responsabilidad. Esta deslegitimación informal de los espacios de cuotas impide que se pueda avanzar en una verdadera igualdad, porque se da una doble estigmatización, por mujer y por dirigente "de cupo".

En este capítulo se señalan las problemáticas que experimentan las mujeres sindicalistas. El techo de cristal en el ascenso laboral se señala como una de las más importantes: en los espacios de socialización informal donde prima la ayuda entre varones las mujeres quedan en desventaja.

El piso pegajoso es una problemática que tiene una gran importancia en la oportunidad de las mujeres para ser responsables sindicales. Esto se debe a que el sindicato es una organización que consume mucho tiempo. Por esto, solo las mujeres que tienen una estructura familiar adecuada y una buena distribución de las tareas domésticas y de cuidado son quienes pueden acceder a los cargos sindicales.

Otra problemática es la legitimidad precaria que poseen como mujeres. Siempre se cuestiona la validez de su puesto, de su capacidad, de su idoneidad, lo que hace que, como ya se mencionó, muchas veces las mujeres no acepten los espacios de cuota. A esto se le suma que el acceso a los puestos de decisión requiere aprender competencias técnicas y de manejo de personas que se adquieren por práctica, a medida que se va ascendiendo en la organización. Las mujeres se ven enclaustradas muchas veces en ciertos cargos como la puesta en marcha de la política de igualdad, y se le dificulta el recorrido típico que permite esta acumulación de recursos.

Como respuesta a algunas de estas problemáticas la autora señala la importancia del "lift as theyrise" que significa que las responsables sindicales a medida que ascienden sindicalmente, hacen subir con ellas a mujeres para darle actividades y responsabilidades. Es una forma de aumentar la cantidad de mujeres en espacios de poder, y también de generar redes de solidaridad femenina.

Para cerrar el capítulo la autora plantea que esta feminización desde arriba es importante pero no suficiente, ya que los mecanismos de discriminación siguen encastrados en el funcionamiento cruzado del mercado de trabajo y el campo sindical. Es por esto que a las mujeres les es difícil en esta situación construir una legitimidad estable y durable. Para solucionar esto se plantea la necesidad de medidas radicales a favor de la igualdad, lo que permite el avance real de las mujeres en lo alto de las organizaciones y la construcción o expresión de formas de solidaridad de género, clase y raza a través de grupos o comisiones no mixtas en diferentes niveles de organización. 
El tercer capítulo es un recorrido por la lucha por la igualdad salarial en el caso británico, y el rol que tuvieron los sindicatos en éste. No sigue con la línea argumental anterior y rompe con el trabajo comparativo que venía haciendo. No obstante creemos que la decisión de incorporarlo al libro es acertada, porque con el tratamiento de otra temática específica del caso británico nos permite, otra vez, volver a hacernos preguntas que pensábamos saldadas. La autora pone en discusión las posturas que muchas veces tuvieron los sindicatos, contrarias a los avances por la igualdad, pero también señala la capacidad de acción y la voluntad de actores varones, quienes se involucraron en la lucha por la igualdad. Ambas menciones dan muestra de cómo las luchas que se dan muchas veces surgen con otras intencionalidades o con actores que en un primer momento no podrían categorizarse como revolucionarios, mientras que al contrario, organizaciones que se supone deberían ser revolucionarias, en este caso los sindicatos, tienen posturas que van en contra de la conquista de derechos.

En este capítulo se pone de relieve la tensión y articulación de formas convencionales de presión y lucha (la negociación colectiva y las formaciones de dirigentes) con formas no convencionales como son las acciones judiciales individuales o colectivas de gran amplitud. En particular, las acciones judiciales para el caso británico son de suma importancia.

Guillaume organiza su exposición en décadas, donde señala la modificación que se da en la estrategia de lucha, con su actor destacado y su forma específica de ser encarada. Plantea como catalizador a la ley de equidad salarial de los 70, que desencadenó un ciclo de huelgas de mujeres en gran Bretaña (entre las que la huelga de Dagenham fue la más destacada). Se reivindicaban la igualdad salarial, la revalorización de los bajos salarios y la implementación de los derechos sociales. A estas huelgas se les suma entre 1975 y 1982 una gran cantidad de denuncias individuales en los tribunales. Estas disminuyen en cantidad a principios de la década del 80 debido a la lentitud del proceso y la baja tasa de éxito.

En la década de los 80 los actores que surgen con más fuerza son las feministas dentro de los sindicatos, quienes se apoyan en la legislación europea para demandar, junto con abogados feministas, enmiendas a la ley de equidad de género, pues la consideraban estrecha. Estos nuevos actores son importantes para la revitalización de las luchas por la igualdad. Como se empiezan a elegir los casos que sean más fáciles de ganar, el aumento de las victorias en los juicios genera que las empresas opten por acordar con los sindicatos la equidad salarial, lo que a su vez genera una nueva dinámica enmarcada en las discusiones entre sindicalistas y empresarios en las negociaciones colectivas.

En 1990 los actores que surgen son los varones sindicalistas que luchaban contra la política de privatización y la degradación del empleo de las mujeres. Son movilizaciones individuales de sindicalistas locales que toman la decisión de sostener y suscitar denuncias grupales de mujeres. El trabajo de concientización e información sobre los derechos de las mujeres que realizan es muy importantes, sin embargo, también se da una utilización pragmática de las denuncias para lograr el aumento de sueldos en épocas que estaban congelados. Por esto la autora explica que en esta época la defensa de la igualdad salarial fue sostenida por razones más pragmáticas que ideológicas, por sindicalistas varones que tomaron riesgos por su propia carrera sindical.

Todo el desarrollo que realiza la autora en este último capítulo, y que pareciera separarse del resto del libro, le permite pensar el compromiso y la importancia de actores críticos tanto al interior como al exterior de las instituciones. El rol destacado de las mujeres de la segunda ola del feminismo y de los varones sindicalistas locales, así como la movilización de aliados en el campo jurídico y político, y a nivel nacional y europeo permite pensar en la heterogeneidad de los actores y sus intereses a la hora de luchar por la igualdad salarial, así como también en el uso pragmático que se le puede dar a la misma.

Por todo lo antes descripto creemos que el libro de Guillaume vuelve a poner en agenda la discusión del sindicalismo en relación con la perspectiva de género y las luchas por la igualdad desde una perspectiva analítica y crítica, basada en un extenso trabajo de campo, que le permite ir más allá de los lugares comunes -en los que muchas veces estos análisis quedan- y pensar realmente cuáles son los resultados de las medidas que se implementan, y qué actores son los que se movilizan y para qué. 
Para pensar el caso argentino, este texto aporta líneas de investigación y preguntas que nos parecen interesantes. En particular, la tensión entre representación estadística y descriptiva, es decir, las políticas aplicadas y la cantidad de mujeres en espacios de decisión. En un contexto en el cual avanzan las políticas de género pero continúan habiendo discriminaciones y dificultades para que las mujeres puedan llegar a los espacios de decisión, nos hace pensar que las políticas de igualdad y las medidas de acción positiva solas no alcanzan. Asimismo, haber tenido mujeres en los más altos puestos de decisión de la región en el último decenio tampoco generó una oleada de medidas y de prácticas que favorecieran el aumento de mujeres en los espacios de poder, con lo cual, como bien dice la autora, ambas son necesarias, aunque no suficientes. Falta todavía la eliminación de los regímenes de desigualdad, esta discriminación reproducida involuntariamente en el interior de las organizaciones y que termina esmerilando los intentos serios por avanzar en la igualdad entre varones y mujeres. Solo con la ruptura de estos mecanismos de discriminación se puede pensar en un verdadero avance hacia la equidad real.

¿Cómo pensar una política integral de género que pueda abarcar todos estos aspectos? ¿Cómo romper con los mecanismos de discriminación más subterráneos en el interior del sindicato y del mundo del trabajo? ¿Cómo articular las políticas de avance de la igualdad con una verdadera democratización de los espacios de poder?

Al leer la obra de Guillaume podemos encontrar una serie de ideas o de pistas a seguir, entre las cuales la más importante es la presión de una masa crítica de mujeres necesaria para lograr cambiar las culturas organizacionales. Y al analizar los desarrollos que el movimiento de mujeres viene generando en la actualidad, nos queda, como cierre, preguntarnos: ¿Está surgiendo y creciendo esa masa crítica necesaria para cambiar las culturas machistas de las organizaciones sindicales?

\section{Notas}

1 La obra de Margaret Maruani es paso obligado si queremos hablar de sindicalismo y género en Francia, y la autora le dedica lo necesario a este trabajo pionero.

2 Confederación Francesa Democrática del Trabajo: la mayor confederación sindical francesa por número de afiliados, y una de las más influyentes, junto a la Confederación General del Trabajo (CGT).

3 Solidaires Unitaires Démocratiques (SUD) grupo de sindicatos con ideología progresista. Una parte se escindió de la CFD en 1988, el resto eran autónomos. Forman parte del Foro Social Europeo y el Foro Social Mundial.

4 Está presente en la mayoría de los sectores industriales y algunos sectores de servicio.

5 El segundo sindicato más grande del Reino Unido. La mayor parte de sus integrantes son del sector público. 